investigating for vomiting and elevated aminotransferase levels in outpatient control. In one of the cases of biliary atresia with microcephaly, deafness and mental-motor retardation was considered due to congenital CMV infection. Other cases were evaluated as perinatally infection. Five patients were given ganciclovir therapy. Duration of the therapy was; six weeks for patient with congenital CMV infection and two weeks for the other four patients.

Conclusion Biliary atresia were seen in all our cases with cholestasis and there is importance of seeing it with CMV hepatitis which, in our conclusion, requires comprehensive studies. In addition, seeing these patient in such a short period of time was found interesting in epidemiological perspective.

\section{P0-0105 EARLY ONSET CONSTIPATION - WHAT ARE THE LESSONS LEARNT?}

F Beal, S Asad, S Mahadevan-Bava. Department of Paediatrics, Russells Hall Hospital, Dudley, Birmingham, UK

\subsection{6/archdischild-2014-307384.774}

Background and aims Constipation developing at $<6$ months of age (early onset constipation-EOC) is common and distressing for infants and carers. It challenges paediatricians, as it may indicate an uncommon but serious, organic cause, especially Hirschsprung's disease (HD).

Aims Evaluate the outcome of EOC and role of rectal biopsy.

Methods We reviewed all children diagnosed as EOC between 2008-2013 referred to the Gastroenterology clinic. We analysed 31 case notes and recorded their demographics, investigations and outcome.

Results Of 31 children, $61 \%$ female, all caucasian except one mixed race. Age of onset reported as since birth in $61 \%$ and 1-5 months in $39 \%$.

$14 / 31$ children were referred for rectal biopsy when clinically suspected to have HD. 3 were positive (all preterm) and 1 anteriorly placed anus missed on routine neonatal examination. The need for further investigation was driven by red flag symptoms. Passing meconium was delayed in only $8 \%$.

Children on hydrolysed milk prior to referral reported no benefit. All were treated with 1/2 laxatives and intermittent suppository.

Over 4-12 months 65\% fully recovered, (4 underwent surgery) and $13 \%$ were controlled with medication.

Conclusion EOC is common and has significant impact on the QoL for infant and carer. No association between delayed meconium and $\mathrm{HD}$ was noted.

The importance of vigilance for diagnosing HD especially in pre-terms must be stressed, also educating trainees about abnormally placed anus. Change in hydrolysed formulae may not be an option but support, advice and medication is the key to success.

\section{PO-0106 SCLEROSING CHOLANGITIS IN CHILDHOOD REPORT OF 3 CASES}

S Ben Ameur, S Alibi, L Sfaihi, M Hsairi, F Kamoun, TH Kamoun, M Hachicha. Pediatics Department, Hedi Chaker Hospital, Sfax, Tunisia

10.1136/archdischild-2014-307384.775

Backgrounds and aims Sclerosing cholangitis (SC) is a chronic cholestatic liver disease characterised by inflammation and progressive bile duct fibrosis. Our purpose was to describe characteristics of SC in childhood.

Methods We performed a retrospective study of 3 childrens with SC followed in the paediatric department of Sfax (20082013).

Results There are 2 boys and 1 girl. The mean age at diagnosis was 6 years. Clinical features at presentation were jaundice (1 case) and hepatosplenomegaly (2 cases). Autoantibodies (antinuclear antibody, smooth muscle antibody, and perinuclear antineutrophil cytoplasmic antibodies) were detected in 1 case. Magnetic resonance cholangiography revealed irregularities with strictures, dilatations and pruning of bile ducts. Histological examination of liver biopsy showed signs of CS stage I (1 case), stage III (1 case) and portal inflammation with infiltration of lymphocytes and plasmocytes and periductal fibrosis (1case).

Search histiocytosis was negative in 3 cases. Colonoscopy with mucosal biopsies revealed no specific inflammatory colitis in one case. The diagnosis of overlap syndrome was made in one case and primary SC (2 cases). All patients were treated with ursodeoxycholic acid and the patient with overlap syndrome received immunosuppressive therapy. After a mean follow-up of 3 years, remission was noted in 2 cases and one patient progressed to cirrhosis and liver failure.

Conclusion SC is a rare cause of chronic cholestasis. Ursodesoxycholic acid is the treatment of choice for all forms of SC but without proof of its effectiveness in preventing progression to secondary biliary cirrhosis.

\section{P0-0107 IMPACT OF GLUTEN FREE DIET ON CLINICAL PROFILES AND ANTHROPOMETRY IN DIFFERENT AGE GROUPS OF CHILDREN WITH CELIAC DISEASE}

${ }^{1} \mathrm{G}$ Dhooria, ${ }^{2} \mathrm{~N}$ Goyal, ${ }^{1} \mathrm{PC}$ Sobti, 'BK Jain. ${ }^{1}$ Pediatrics, Dayanand Medical College and Hospital, Ludhiana, India; ${ }^{2}$ Pediatrics, Deep Hospital, Ludhiana, India

\subsection{6/archdischild-2014-307384.776}

Objective To evaluate impact of gluten free diet in children with celiac disease in different age groups on clinical profile and anthropometric measurements.

Design A prospective interventional study.

Methods Twenty five of the 32 children diagnosed as having celiac disease on the basis of positive anti-tissue transglutaminase IgA (tTG) and duodenal biopsy were included. Patients underwent a clinical examination and anthropometry measurements at diagnosis, 3 months and 6 months of strict gluten free diet.

Results There was a dramatic clinical improvement in symptomology in all cases on institution of gluten free diet at 6 months of follow up. Loose motions, abdominal pain, irritability disappeared in all patients except two children had anaemia $(8 \%)$ and one child had abdominal distension (4\%). Significant weight and height gain was noted in children after initiation of gluten free diet. The mean weight and height increased significantly after 3 and 6 months of gluten free diet $(\mathrm{p}<0.0001)$. Also at 6 months of gluten free diet $86 \%$ of the children $<5$ years and $50 \%$ of the children between 5-10 years of age gained weight upto $>80 \%$ of expected weight whereas none of the children $>10$ years of age reached that level. Similarly, all the children $<5$ years achieved normal height while only $33 \%$ of the other children attained normal height after 6 months of gluten free diet.

Conclusion Early diagnosis and institution of strict gluten free diet is required to gain maximum growth potential in children with celiac disease. 